Journal of Materials Science (2011) 46:2838-2842

\title{
Electropulse-induced cementite nanoparticle formation in deformed pearlitic steels
}

\author{
R.S. Qin ${ }^{1 *}$, E.I. Samuel ${ }^{2}$ and A. Bhowmik ${ }^{2}$
}

1. Department of Materials, Imperial College London, Exhibition Road, London SW7 2AZ, United

Kingdom

2. Graduate Institute of Ferrous Technology, Pohang University of Science and Technology, Pohang 790-784, South Korea

\begin{abstract}
Passing one electric-current pulse through deformed pearlitic steel wires at room temperature causes the formation of cementite particles around $30 \mathrm{~nm}$ in size. This is found not only in some particular locations but throughout the cementite area, which reveals a different mechanism from traditional spheroidization of cementite plates because the latter leads to the formation of particles with much large size. Transmission electron microscopy images show electropulse-induced strain relief and formation of fine precipitations. Differential scanning calorimetry analysis demonstrates the additional stored free energy by electropulsing treatment. The raised free energy accounts the increased interface area in finer microstructure of materials. The experiment evidences that the passing electric current in metal has alternated the free energy sequence of various microstructures in comparison with that of current-free system.
\end{abstract}

Keywords: Steels; Microstructure; Electrical conductivity; Electropulse

*Corresponding author. Tel: +44 207594 6803; fax: +44 2075946757.

Email address: r.qin@imperial.ac.uk 


\section{Introduction}

When metals carry electric current, their system free energy encloses an additional term $G^{e}$ in comparison with the system without electric current but at the same state (constitution, temperature, pressure etc.). The method for computing $G^{e}$ is derived from Maxwell Faraday equation and Maxwell Ampere law, and is presented in two different formats. One is to integrate the product of magnetic field $\vec{B}$ and magnetizing field $\vec{H}$ throughout the space

$$
G^{e}=-\frac{1}{2} \int \vec{B} \cdot \vec{H} d r
$$

where $r$ is a point in real space. Another format is to integrate the product of current density $\vec{j}$ and magnetic vector potential $\vec{A}$ inside conductive materials because $|\vec{j}|=0$ outside.

$$
G^{e}=-\frac{1}{2} \int \vec{j} \cdot \vec{A} d r
$$

Using the general solution of $\vec{A}$ in given current distribution $\vec{j}$, Eq. (2) can be represented by

$$
G^{e}=-\frac{\mu}{8 \pi} \iint \frac{\vec{j}(r) \cdot \vec{j}\left(r^{\prime}\right)}{\left|r-r^{\prime}\right|} d r d r^{\prime}
$$

where $\mu$ is the magnetic permeability. $r$ and $r^{\prime}$ are two different positions in metal, respectively. The reference state for Eq. (3) is at the infinite far away location.

In microstructure transformation, which can be due to phase transition or structural relaxation, electrical conductivity of part or whole metal changes from one value to another. This causes the

alternation of current distribution from $\vec{j}_{0}$ to $\vec{j}_{1}$, and hence the change of electric-current associated free energy $\Delta G^{e}$. Using Eq. (3), it has 


$$
\Delta G^{e}=\frac{\mu}{8 \pi} \iint \frac{\vec{j}_{0}(r) \vec{j}_{0}\left(r^{\prime}\right)-\vec{j}_{1}(r) \vec{j}_{1}\left(r^{\prime}\right)}{\left|r-r^{\prime}\right|} d^{3} r d^{3} r^{\prime}
$$

where the sub-index 0 and 1 represent the states before and after microstructural transformation. Eq. (4) shows that the passing electric current in metal affects thermodynamics of phase transformation and tends to generate the most negative $\Delta G^{e}$.

Quantitative calculation of $\Delta G^{e}$ by Eq. (4) has been performed in nucleation [1], nanoscale crystal growth [2], microstructure damage recovery [3] and microscale crack healing [4]. It has been found that the free energy associated with electric current, $\Delta G^{e}$, plays an important role when the current density surpasses $10^{3} \mathrm{~A} / \mathrm{cm}^{2}$. The calculation explains the experimental observations in solidification [5, 6], amorphous alloy crystallization [7], crack healing in steels [8], solid phase transition [9, 10] and so on. Dolinsky and Elperin have applied Eq. (4) to conductor explosion [11] and solid-gas transition $[12,13]$. The general conclusion after all of those calculations is that the passing electric current promotes the formation and growth of phase with higher electrical conductivity. Kinetically, it has been found that passing electric current can accelerate microstructure transformation [7, 14] and decrease the transition starting temperature [15-17]. Recently, Samuel et al reported the electropulsing-accelerated spheroidization of cementite plates in deformed pearlitic steels [18]. Both the thermodynamic and kinetic effects are proved different from ohmic heating and percolation. The application of pulsed electric current is to reduce the ohmic heating effect.

Cold plastic deformation changes the electric conductivity of metals [19]. This is due to the change of microstructure of materials such as the generation and accumulation of dislocations, change of total amount of interface and grain edge [20], the stored strain and so on. The deformed 
microstructure is frequently at non-equilibrium state, but is unable to transform into the equilibrium state due to the kinetic barrier at low temperature. This situation provides with another ideal opportunity to study the effect of electropulse on microstructure transformation. From industrial point of view, the cold worked metals have many properties to improve. Exploration of the effect of electropulse on deformed metals is meaningful.

\section{Experimental procedure}

Commercial steel wires of chemical composition Fe-0.8C-0.2Si-0.5Mn (in weight \%) were received, heat treated to form full pearlitic microstructure, and then cold-drawn to a true strain of 1.61 and cut into $30 \mathrm{~mm}$ length samples. A number of samples were put into the in-house designed electropulsing equipment for treatment, and their microstructures are examined and compared with those without electropulsing samples. The electric current pulse is damped-sinusoidal waveform. The highest peak density is $9.61 \times 10^{9} \mathrm{~A} / \mathrm{m}^{2}$. The first half-wave lasted $150 \mu$ s and the signal disappearing completely after $1800 \mu \mathrm{s}$ due to damping. Only one electropulse was applied to the samples. Both the electropulsed and un-electropulsed samples were prepared by cutting along the longitudinal and cross sections, polished and etched in natal for metallographic analysis and sent to Carl Zeiss made

Ultra-55 scanning electron microscope (SEM), JOEL made 2100F transmission electron microscope (TEM) and Netzsch STA-449C differential calorimetry for examination. The Vickers hardness was measured using a WOLPERT-425 SVD machine (UK Calibrations Ltd., Lancashire, UK) employing $10-\mathrm{kg}$ load for a dwell time of $10 \mathrm{~s}$

\section{Results and Discussion}


Fig. 1 demonstrates the effect of electropulse on microstructures transformation of deformed pearlitic steel by SEM micrographs, where Fig. 1(a) is the microstructure before electropulse and Fig. 1(b) is the microstructure after an electropulse. The bright plates are cementite. Pearlite consists of alternating lamellae of cementite and ferrite but the latter is easier to dissolve in etching liquid because of its iron-rich composition. The images were taken from the longitudinal cut section. Fig. 1(a) shows the elongated but integrated cementite plates. In Fig. 1(b), the cementite plates are fragmented into fine particles of around $30 \mathrm{~nm}$ in size and distributed homogeneously along the original cementite plates. This fragmentation is purely due to the effect of electropulse because the only different processing between two samples is the electropulse treatment.


Figure 1 SEM micrographs of electropulse-induced microstructure transformation in pearlitic steel (a) before electropulse and (b) after electropulse.

As is known from literature, cementite plates in pearlitic steels can be fragmented into small parts and spheroidized under static annealing at temperature above $580{ }^{\circ} \mathrm{C}$ for a few hours [21]. The fragments are observed in an aspect ratio between 6:1 and 8:1, and the pre-processing by various deformations and annealing conditions did not change the aspect ratio [22]. The spheroidization is to 
reduce the total area of interface and hence to minimize the system free energy. A simple mathematic calculation reveals that the minimum average diameter of those spherical particles by annealing is three times of the thickness of the original plate. The volume of a particle with the minimum average diameter corresponds to a plate-shape fragment with aspect ratio 3.759:1. In observation of Fig. 1(b), it can be found that the particle size obtained by electropulse processing is smaller than the theoretical minimum particle size in annealing. This means that the passing electric current provided with extra free energy during the microstructure transformation and the latter enabled to generate extra interface area so that the particle size can achieve much finer than in thermal annealing. The extra free energy is provided by the electric-current associated free energy. This falls into the scope of Eq. (4) if the new phase is with higher electrical conductivity. Reduction of dislocation density and lattice distortion leads to the increase of electrical conductivity in most of the cases [23].

The transmission electron micrographs are demonstrated in Fig. 2, where Fig. 2(a) is for the sample before electropulsing treatment and Fig. 2(b) is for the sample after electropulsing. Both are from cross sectional view. Fig 2(a) shows many dark fields in the noticeable white background of the ferrite matrix. Those dark fields are strain fields and contribute to the change of electric conductivity. Our measurement shows that the bulk electric resistivity of un-electropulsed sample is $2.14 \times 10^{-7}$ $\Omega \cdot \mathrm{m}$, while that of the electropulsed sample is $1.64 \times 10^{-7} \Omega \cdot \mathrm{m}$. This is in alongside the theoretical conclusion of that the high conductive phase is promoted to form on application of electropulse.

Those noticeable contrast strain areas are disappeared in Fig. 2(b). This evidences the electropulseinduced structural relaxation. Fig. 2(b) also shows some very fine precipitates on the surface of the 
lamellar structure, as highlighted by arrows. This is different from observations in the cementite spheroidization observed and described in isothermal annealing processes.
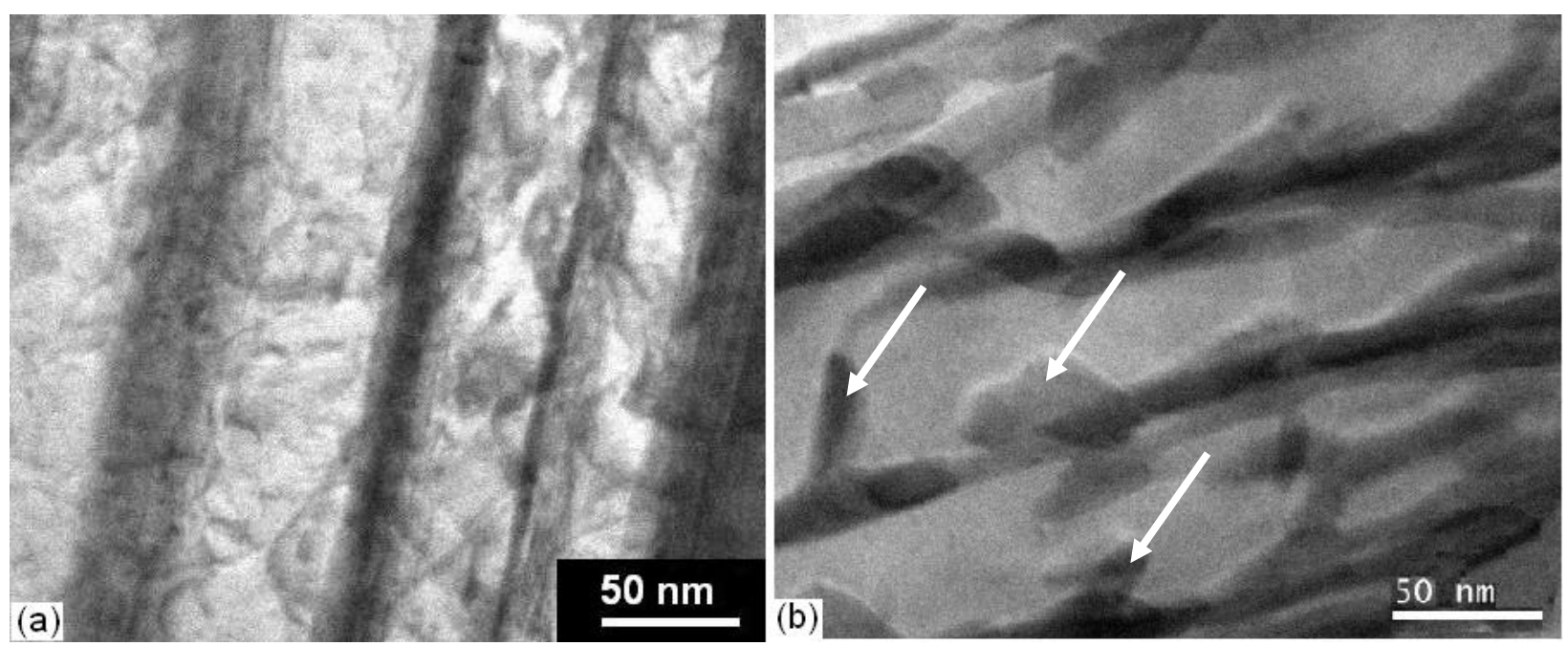

Figure 2. Transmission electron micrographs in cross sectional view. (a) Before electropulsing; (b) After electropulsing.

Figure 3 shows the heat flow recorded using differential scanning calorimeter during (a) heating and (b) cooling cycles for the steel before (solid line) and after (dashed line) electropulsing. It can be seen clearly from Fig 3(a) that the area under the dashed line is larger than that of under solid line. This implies that the electropulsed sample has released more heat than the un-electropulsed sample during the DSC experiment. This means that the electropulsed sample is at higher free energy state in comparison with the sample without electropulsing treatment. Obviously, part of the excess energy is stored as the increased area of interface as demonstrated in Fig. 1(b). This is in agreement with the reported increase in free energy in plain carbon steel alloyed with $\mathrm{Co}$, in which it was rationalized by the increase in ferrite cementite interfacial area [24]. The excess free energy, however, has been 
released at austenitizing temperature due to the lost of electropulse-induced structure. In the DSC cooling stage, both the electropulsed and un-electropulsed show that same behaviors, as illustrated in Fig 3(b). This is due the similar microstructure in both samples after austenitizing temperature.
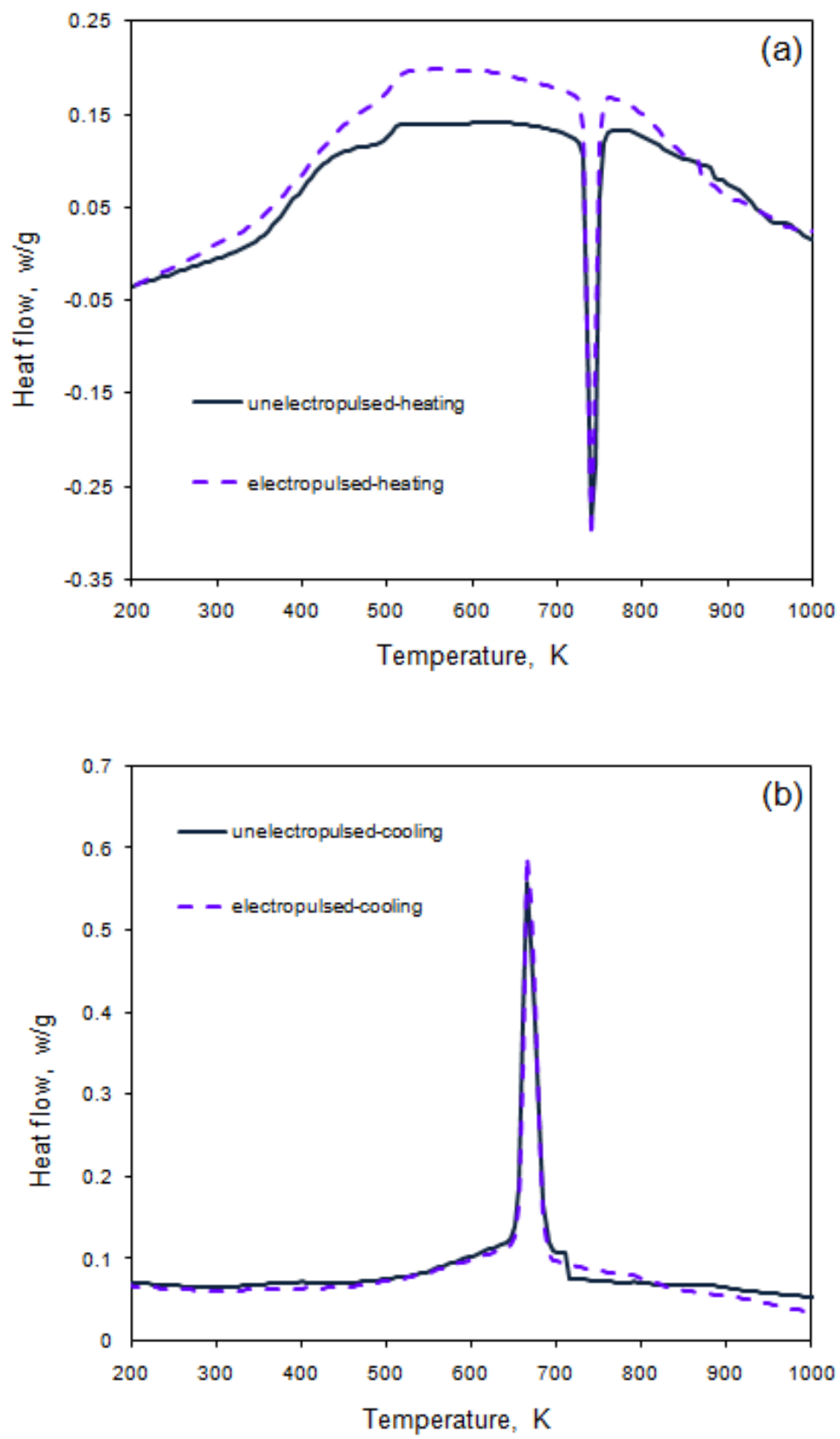

Fig. 3 Differential scanning calorimetric recordings for the cold-deformed pearlite before and after application of a pulse for the heating cycle. (a) heating (b) cooling. 
The Vicker's hardness value of the samples before electropulsing was $415 \mathrm{HV}_{10}$ while after electropulsing is $460 \mathrm{HV}_{10}$. The increment of hardness reflects the effect of formation of finer grains in sample, which agrees with microstructure observation. It is worth to point out that heat treatment of drawn steel wire usually leads to the decrease of hardness due to the annihilation of dislocation under heat treatment.

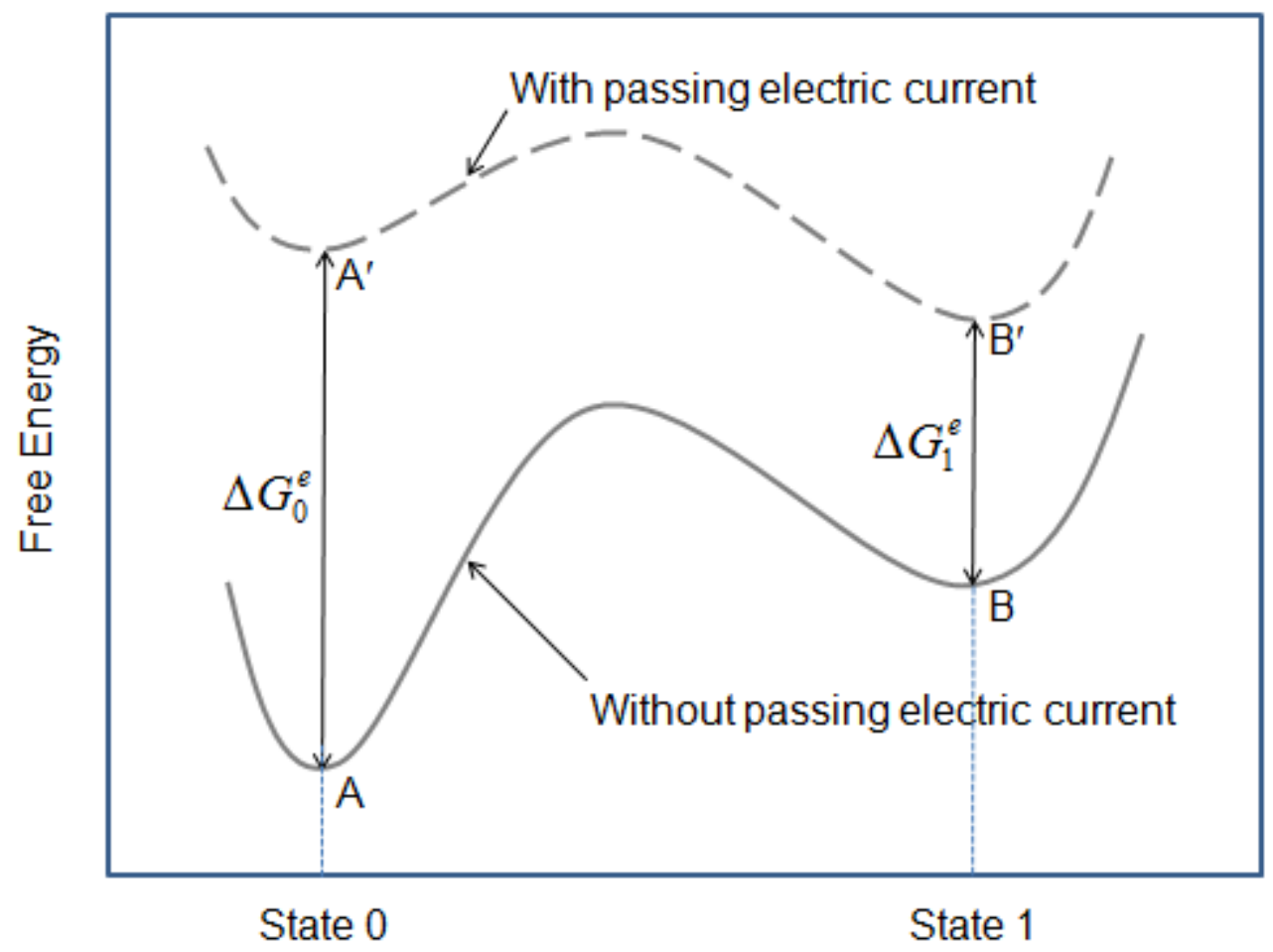

Fig. 4 Schematic diagram of the mechanism of electropulse induced microstructure transformation.

To demonstrate the mechanism of electropulse-induced microstructure transformation more clearly, the process is schematically presented in Fig. 4 . The state 0 represents the steel with microstructure before transformation and the state 1 represents the microstructure after transformation. Without the passing electric current, the free energy in state 1 (denoted by B) is higher than that of the state 0 (denoted by A). This is not possible to happen because the second law of thermodynamics is violated. 
When the electric current is passing through the steel, however, the free energy of state 0 is change into the position denoted as A' and that of the state 1 is changed into the position denoted as B'. The difference of free energy increasing for both states is due to their different electrical conductivity, i.e. $\Delta G_{o}^{e}>\Delta G_{1}^{e}$. In this situation, the free energy of state 0 is higher than the free energy of state 1. Microstructure transformation happens during the passing of electric current. After electric current passing by, state 1 is left at high free energy state but unable to transform into the lower free energy state 0 because of the high kinetic barrier at low temperature. The kinetic barrier is much lower during the passing of high density electric current according to the previous experimental observations [14-17].

The unique effect of electropulse provides kinetic possibility for the observed transformation. On the one hand, the high intensity electric current pulse causes rapid increase of the temperature in steel wire. This introduces a huge amount of vacancies along grain boundary, interface and dislocations. Those vacancies migrate into cementite plates and enhance the diffusion of elements. The cementite plates are therefore able to transform into spherical particles [18]. However, one another hand, due to the short duration nature of electropulse the temperature drops into room temperature quickly and the kinetic barrier prevented further diffusion after electropulse. The nanoparticles are unable to grow larger.

\section{Conclusions}

(1) Passing one electric current pulse with peak density $9.61 \times 10^{9} \mathrm{~A} / \mathrm{m}^{2}$ and pulse duration less than 1 ms through deformed pearlitic steel causes the formation of nanoscale cementite particles around 
$30 \mathrm{~nm}$ in size. This transformation is found throughout the sample. The particle size is smaller than the minimum theoretical value obtained by free energy estimation in thermal annealing treatment, shows the different mechanism of microstructural transformation driven by passing electric current in metals.

(2) The passing electric current has caused the structural relaxation. The strain area formed in cold worked metal is disappeared after electropulse. The bulk electrical resistivity of pearlitic steel is

reduced from $2.14 \times 10^{-7} \Omega \cdot \mathrm{m}$ to $1.64 \times 10^{-7} \Omega \cdot \mathrm{m}$ after electropulsing treatment. This gives good agreement with the theoretical prediction of that the passing electric current promotes the formation and growth of phases with high conductivity.

(3) The passing electric current in steels changes the free energy sequence of various phases, and can cause the microstructure transformation which is not possible in the same system but without electric current. The low temperature processing allows the retaining of the electropulse-induced microstructure.

\section{Acknowledgements}

The authors are grateful to Professor HKDH Bhadeshia for encourage and constructive discussion during the research. RQ is grateful to Professors N. Alford and W. Lee for the provision of laboratory facilities at Imperial College London, and to Corus and Royal Academy of Engineering for financial support of his work. ES and AB are grateful to GIFT and POSCO for financial supports. 


\section{References}

[1] Qin RS, Zhou BL (1998) Int J Non-equilib Proc 11:77

[2] Qin RS, Su SX, Guo JD, He GH, Zhou BL (1998) Nanostruc Mater 10:71

[3] Qin RS, Su SX, Guo JD, He GH, Zhou BL (1996) Biomimetics 4:121

[4] Qin RS, Su SX (2002) J Mater Res 17:2048

[5] Conrad H (2000) Mater Sci Eng A 287:205

[6] Barnak J, Sprecher AFS, Conrad H (1995) Scripta Metall Mater 32:879

[7] Mizubayashi H, Okuda S (1989) Phys Rev B 40:8057

[8] Zhou YZ, Qin RS, Xiao SH, He GH, Zhou BL (2000) J Mater Res 15:1056

[9] Zhang W, Zhao WS, Li DX, Sui ML (2004) Appl Phys Lett 84:4872

[10] Wang XL, Guo JD, Wang YM, Wu XY, Wang BQ (2006) Appl Phys Lett 89:061910

[11] Dolinsky Y, Elperin T (1993) Appl Phys 73:5283

[12] Dolinsky Y, Elperin T (1993) Phys Rev B 47:14778

[13] Dolinsky Y, Elperin T (1994) Phys Rev B 50:52

[14] Hao T, Tanimoto H, Mizubayashi H (2005) Mater Trans 46:2898

[15] Teng GQ, Chao YS, Lai ZH, Dong L (1994) Chinese Sci Bull 39:974

[16] Takemoto R, Mizubayashi H (1995) Acta Metall Mater 43:1495

[17] Takemoto R, Nagata M, Mizubayashi H (1996) Acta Metall Mater 44:2787

[18] Samuel EI, Bhowmik A, Qin RS (2010) J Mater Res 25:1020.

[19] Çetinarslan CS (2009) Mater Design 30:671

[20] Chae JY, Qin RS, Bhadeshia HKDH (2009) ISIJ International 49:115

[21] Chattopadhyay S, Sellars CM (1977) Metallography 10:89

[22] Chattopadhyay S, Sellars CM (1985) Acta Metall 30:157

[23] Qin RS and Zhou BL (1996) Nanostruct Mater 7:741 
[24] Hagel WC, Pound GM, Mehl RF (1956) Acta Metall 4:37 